Muséologies

Les cahiers d'études supérieures

muséologies

\title{
Tous différents, tous égaux, sous le signe de l'hospitalité
}

\section{Margaret Pfenninger}

Volume 3, numéro 2, printemps 2009

URI : https://id.erudit.org/iderudit/1033562ar

DOI : https://doi.org/10.7202/1033562ar

Aller au sommaire du numéro

Éditeur(s)

Association Québécoise de Promotion des Recherches Étudiantes en Muséologie (AQPREM)

ISSN

1718-5181 (imprimé)

1929-7815 (numérique)

Découvrir la revue

Citer cet article

Pfenninger, M. (2009). Tous différents, tous égaux, sous le signe de l'hospitalité Muséologies, 3(2), 64-75. https://doi.org/10.7202/1033562ar d'utilisation que vous pouvez consulter en ligne.

https://apropos.erudit.org/fr/usagers/politique-dutilisation/ 
Article quatre

\section{Tous différents, tous égaux,}

\section{sous le signe de l'hospitalité}

MARGARET PFENNINGER EST CONSERVATEUR, RESPONSABLE DU SERVICE ÉDUCATIF DES MUSÉES DE STRASBOURG. APRĖS UNE PREMIÈRE EXPÉRIENCE AUXÉTATS-UNIS, AU DÉPARTEMENT D'ÉDUCATION DE LINDIANAPOLIS MUSEUM OF ART, ELIE TRAVAILLE POUR LE SERVICE ÉDUCATIF DESMUSÉES DE LA VILLEDE STRASBOURG DEPUIS LA FINDES ANNÉES SOIXANTE-DIX. SPÉCIALISÉE EN ART MODERNE, ELLEEST COMMISSAIRE DE LEXXPOSITION CHROMAMIX 2 QUI A LIEU ACTUELLEMENT AU MUSÉE D'ART MODERNE ET CONTEMPORAN JUSQUAU 15 NOVEMBRE 2009. REALISEEE À PARTIR DUNE SÉLECTION DCEUVRES DES DIX MUSÉES DE STRASBOURG SUR LE THEME DE LA COULEUR, CETTE EXPOSTTION SE VEUT UN VÉRITABLE MOYEN DE MÉDIATION OU LA CONVIVIALITÉ EST LE FZL CONDUCTEUR.

[margaretpfenninger@cus-strasbourg.net] 
Invitée à présenter l'organisation d'un réseau de musées en tant que responsable d'un des services transversaux des musées, je trouve un peu gênant le fait de présenter ce qui à notre avis est loin d'être abouti sur le plan de l'accueil des visiteurs individuels dans les dix musées de Strasbourg [1]. Comme pour beaucoup de musées français hors Paris, une réelle politique d'accompagnement des visiteurs individuels a du mal à capitaliser les acquis accumulés à la suite d'une longue expérience de l'accueil direct des groupes de tous horizons. Ce qui suit est un résumé des recherches que nous y effectuons ainsi que quelques indications de pistes que nous souhaitons approfondir dans un proche avenir.

\section{Tous différents, tous égaux}

Un service éducatif commun à un réseau de dix musées est une situation " avantageusement inconfortable». Avantageuse car nous bénéficions d'une équipe de médiation avec des spécialisations diversifiées (archéologues, historiens, historiens d'art, artistes) pour mettre en place une politique d'accueil innovante, fondée sur l'étude approfondie des différentes collections. Mais inconfortable car nous n'avons jamais assez de moyens (temps, personnel) pour mener à bien un accompagnement accompli dans l'ensemble des musées de façon identique. Les conservateurs nous font souvent remarquer la différence entre le nombre de prestations que nous proposons par musée. 
(les appartements des cardinaux de Rohan et les collections d'arts décoratifs strasbourgeois de 1681 au milieu du XIXe siècle),

le Musée archéologique

(collection régionale de la préhistoire à l'aube du Moyen Âge)

le Musée des beaux-arts (la peinture européenne du Moyen Âge à 1870), le Musée de l'Euvre Notre-Dame

(sept siècles d'art à Strasbourg et dans la région du Rhin supérieur), le Musée alsacien (arts et traditions populaires de la vie rurale en Alsace aux

$X V \mid \|^{e}$ et $X \mid X^{e}$ siècles), le Musée historique

(histoire politique, économique et sociale de la ville de Strasbourg),

le Musée Tomi Ungerer

(collection de dessins de Tomi Ungerer,

dessinateur et illustrateur),

le Musée d'art moderne et contemporain (l'art européen occidental de 1870 à nos jours),

le Cabinet des estampes

et des dessins

(ensemble couvrant cinq siècles, notamment dans les domaines des beaux-arts et des arts décoratifs) et le Musée zoologique (panorama de la diversité animale).
Inconfortable aussi car nous devons adhérer à une politique globale tout en l'adaptant à chaque situation muséale spécifique. Nous dépendons directement du directeur du réseau des musées, mais nous collaborons aussi directement avec chacun des conservateurs, en fonction des particularités de chaque collection.

Au fil des années, nous avons mis au point différentes façons de travailler en réseau:

- mise en place d'une politique des publics inter-musées par l'intermédiaire de relais communs (scolaire, handicap, famille...);

- développement de partenaires culturels qui interviennent régulièrement dans les différents musées selon un programme trimestriel commun;

- organisation d'expositions tous publics à partir des œuvres de l'ensemble des collections;

- et, avant tout, mise en place, malgré nos différences, surtout grâce à elles, d'une philosophie commune à l'ensemble de nos actions dans l'ensemble des musées (voir plus loin).

En préparation à l'arrivée de la nouvelle directrice des Musées de Strasbourg en mars 2007, nous avons proposé (fin 2006) à tous les conservateurs, chefs d'établissement, un questionnaire/audit (voir annexe) dans le but de connaître leurs attentes en matière d'accueil des visiteurs. Avant tout nous souhaitions amorcer avec eux une réflexion sur une politique des publics commune et sur comment nous pourrions mieux travailler ensemble. Questionnaire en main, nous avons ainsi passé au moins deux heures de discussion et d'échanges extrêmement enrichissants avec chacun des conservateurs.

La majorité des conservateurs s'accordaient sur la plupart des questions d'ordre logistique ou opérationnel. Ils souhaitaient maintenir un seul service éducatif (ou service des publics) pour l'ensemble des musées de Strasbourg ainsi que l'accueil des scolaires et des responsables de groupes. Pratiquement tous voulaient aussi que nous approfondissions le travail entrepris avec les personnes en situation de handicap et en insertion et que nous continuions à participer à la rédaction des différents documents d'aide à la visite pour tous les publics. Chaque conservateur avait par contre une manière très personnelle de voir, d'imaginer son musée et donc de comprendre notre rôle en son sein. Ils avaient envie que le travail avec les publics soit maintenu, voire augmenté, mais nous avons constaté qu'ils n'avaient pas du tout la même manière d'envisager cette collaboration. Ils souhaitaient une participation engagée de notre part, mais cette collaboration ne pouvait pas être menée à l'identique dans tous les musées, ni au même rythme. 
Notre défi était donc double: accomplir un travail juste et pertinent de façon équilatérale dans chaque musée tout en répondant aux exigences de chaque conservateur et en tenant compte des besoins spécifiques des visiteurs, et ce, sans moyens supplémentaires.

Afin que la multiplicité des musées ne soit pas un frein mais au contraire un moteur, nous nous sommes penchés sur le célèbre slogan d'Amnistie internationale, "Tous différents, tous égaux », où l'égalité s'affirme par la manifestation des différences. Pour les dix musées de Strasbourg, cela ne voulait pas dire que notre intervention devait être équivalente dans chaque musée pour qu'ils soient égaux, qu'il fallait organiser le même nombre d'activités et proposer les mêmes types de prestations. Au contraire, c'est plutôt en favorisant ce qui rend chaque musée différent qu'ils deviennent enfin égaux. Les particularités des collections et les bâtiments qui les abritent doivent bien entendu toujours nous guider, mais il faut surtout trouver les moyens pour que la vision de chaque conservateur par rapport à son propre musée puisse devenir une force qui motive de nouvelles manières de recevoir et d'accompagner les visiteurs dans chaque musée, bref, qui aide à faire de chaque visite dans chaque musée une expérience unique et singulière.

Malgré leurs différences fondamentales, nous avons tout de même réussi à déterminer trois types de musées, aboutissant ainsi à une structure opérationnelle qui aide à mieux répondre aux besoins en termes d'accueil des visiteurs. Il est intéressant de constater que ce n'est pas le contenu scientifique de leurs collections qui les rapproche, mais la façon dont les conservateurs envisagent leur musée et l'accueil subséquent des visiteurs.

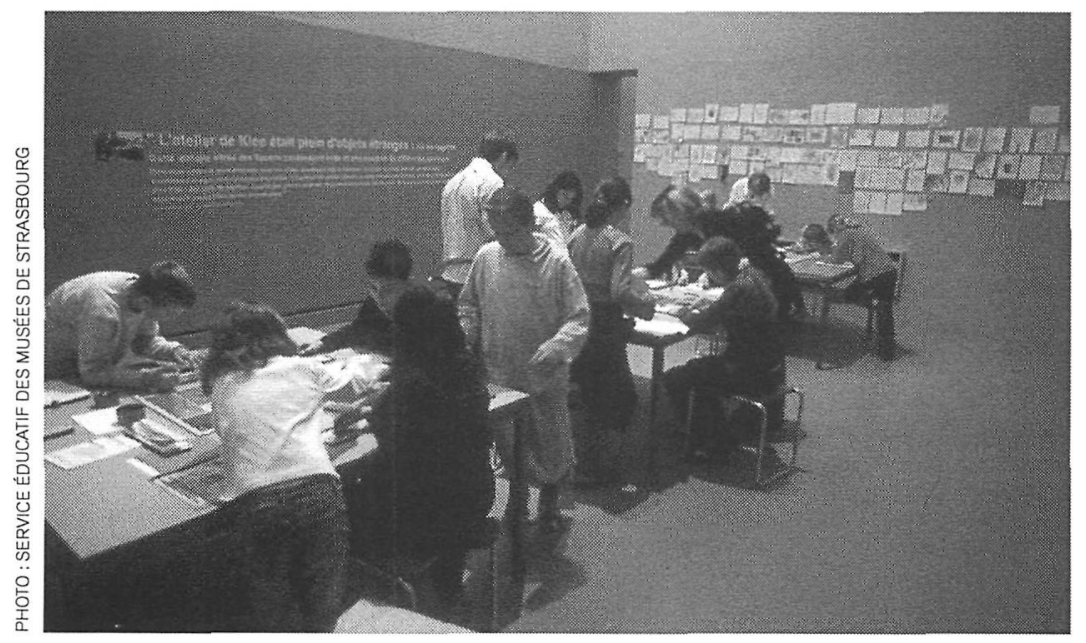

3 tables thématiques en libre accès contenant de petits exercices pour expérimenter les notions clefs relevées dans l'exposition et tirées de l'enseignement de Paul Klee au Bauhaus dans un atelier situé en aval de l'exposition Paul Klee et la nature de l'art. 
Voici ces trois types:

\section{TYPE 1}

Musées comme lieux de vie

$\begin{array}{ll}\text { MUSÉES CONCERNÉS } & \text { - Musée de l'CEuvre Notre-Dame } \\ & \text { - Musée zoologique } \\ \text { - Musée d'art moderne et contemporain } & \\ \text { CARACTÉRISTIQUES } & \text { - Musées assez vastes } \\ \text { MOTS COMMUNS UTILISÉS } & \text { - Convivial } \\ \text { POUR DÉCRIRE COMMENT } & \text { - Ouvert } \\ \text { LES CONSERVATEURS SOU- } & \text { - Lieu de rencontres } \\ \text { HAITENT QUE LES VISITEURS } & \\ \text { PERÇOIVENT LEUR MUSÉE } & \\ \text { TYPES D'ACTIVITÉS } & \text { - Les rencontres entre les visiteurs } \\ \text { À PRIVILÉGIER } & \text { devant les œuvres } \\ \text { PUBLICS À PRIVILÉGIER } & \text { - Les activités pour les groupes et les individuels } \\ & \text { pour le public en famille }\end{array}$

TYPE 2

Musées comme lieux de délectation individuelle

MUSÉES CONCERNÉS

CARACTÉRISTIQUES

MOTS COMMUNS UTILISÉS POUR DÉCRIRE COMMENT LES CONSERVATEURS SOUHAITENT QUE LES VISITEURS PERÇOIVENT LEUR MUSÉE

TYPES D'ACTIVITÉS

À PRIVILÉGIER
- Musée des arts décoratifs

- Musée Tomi Ungerer

- Musée des beaux-arts

- Musées plus petits, difficile de concilier à la fois visiteurs individuels et groupes

- Esthétique

- Lieu de contemplation
- Les dispositifs qui sollicitent la rencontre individuelle des collections

- Les conservateurs souhaitent privilégier plutôt un espace muséal calme parce que cela favorise la compréhension et l'appréhension des collections

- Avant tout visiteurs individuels adultes 
TYPE 3

Musées comme point de départ ou point de chute d'une approche

citoyenne engagée

$\begin{array}{ll}\text { MUSÉES CONCERNÉS } & \text { - Musée historique } \\ & \text { - Musée alsacien } \\ & \text { - Musée archéologique } \\ \text { CARACTÉRISTIQUES } & \text { Musées petits et moyens pouvant accueillir } \\ & \text { à la fois groupes et individus } \\ \text { MOTS COMMUNS UTILISÉS } & \text { - L'accent est mis sur le lien avec la vie } \\ \text { POUR DÉCRIRE COMMENT } & \text { quotidienne / la ville } \\ \text { LES CONSERVATEURS SOU- } & \text { - L'ouverture sur des points de vue différents } \\ \text { HAITENT QUE LES VISITEURS } & \text { suivant les époques } \\ \text { PERÇOIVENT LEUR MUSÉE } & \\ \text { TYPES D'ACTIVITÉS } & \text { Tout ce qui peut aider les visiteurs à mieux } \\ \text { À PRIVILÉGIER } & \text { Ouvrir les thématiques des collections vers } \\ \text { PUBLICS À PRIVILÉGIER } & \text { la vie quotidienne des visiteurs } \\ & \text { Tous publics }\end{array}$

Voici quelques exemples non exhaustifs de pistes, que nous explorons ou que nous projetons explorer pour amorcer une nouvelle politique d'accueil différenciée pour chaque type de musée:

\begin{abstract}
Musée historique : un musée pour une approche active citoyenne Privilégier des visites actives de la ville selon les thématiques que les visiteurs peuvent retrouver (en amont ou aval) dans les collections, par le biais des parcours urbains pour les visiteurs individuels, des dépliants gratuits pour les jeunes, des carnets de voyage pour les classes, adaptables à tous les quartiers.
\end{abstract}

\title{
Musée des beaux-arts : un musée de délectation individuelle
}

Proposer dans les salles des fiches orientées vers la muséographie que le conservateur souhaite mettre de l'avant lors de la visite. Repenser la salle des publics où les visiteurs pourraient approfondir leurs connaissances, découvrir d'autres artistes et d'autres œuvres, construire des parcours thématiques, explorer les composantes de la muséographie. 


\section{Musée de l'Guvre Notre-Dame : un musée lieu de vie}

Participer activement avec le conservateur au projet d'extension où sont à l'étude, entres autres, une salle d'introduction pour les groupes et une salle des publics au sein des collections pour les visiteurs individuels, enfants et adultes.

\section{Sous le signe de l'hospitalité}

Cherchant à créer une synergie entre ces recherches spécifiques, le service éducatif des musées de Strasbourg s'appuie sur un principe de base commun à partir duquel nous essayons de fonder et de tester nos différentes actions. Une sorte de pierre de touche nous aidant à répondre avec justesse aux différentes situations.

À l'instar des artistes qui intègrent intimement la notion de convivialité dans leur processus de création, nous travaillons depuis quelques années sur la manière d'intégrer la notion d'hospitalité dans son acception la plus large dans l'ensemble de nos actions, acception qui touche plusieurs facettes aux dimensions variables. En voici brièvement quelques-unes que nous explorons actuellement.

Un des aspects fondamentaux de la notion del'hospitalité est la dimension humaine, intime et personnelle exigée de chaque médiateur dans son rôle d'accueillant au musée. Selon la sociologue Anne Gotman, pratiquer l'hospitalité exige nécessairement une ouverture à l'Autre, une reconnaissance de l'Autre et de sa différence. C'est une pratique qui nous renseigne aussi et avant tout sur notre propre rapport à l'Autre ${ }^{[2]}$.

Nous nous intéressons également à la dimension interculturelle de l'hospitalité qui donne des éclairages sur les modes de vie et de pensées des différentes communautés d'esprit de nos visiteurs. Au service éducatif nous voulons nous inspirer de l'hospitalité des démarches artistiques, mais aussi des cultures anciennes et traditionnelles, qui comprennent des rites dans le processus d'accueil, entre celui qui reçoit et celui qui est reçu. En fonction de sa culture personnelle et de la culture de l'Autre, où les deux rôles d' " hôte " sont pleinement explorés, il s'agit de savoir recevoir, respecter l'Autre, c'est-à-dire accepter, apprécier, goûter les dons qu'apportent les visiteurs. Il faut en outre savoir donner, offrir et partager les dons de chaque musée (éclairages historiques, clés de lecture des œuvres d'art, savoir faire techniques, etc.) pour qu'ils soient accessibles à tous, pendant les trois temps symboliques de l'hospitalité: l'accueil, le séjour et «l'échange des cadeaux ", le départ ${ }^{[3]}$. 
Étudier l'hospitalité comprend aussi traditionnellement un travail sur un lieu lui-même, qui au départ est étranger, voire hostile, pour qu'il devienne hospice ou abri pour ceux qui y sont accueillis. Un ensemble de règles partagées est souvent établi, garant de meilleurs usages des lieux, voire d'une cohabitation réussie. En ce qui concerne les musées, si nous essayons d'être plus attentifs à la liberté que chaque visiteur se doit d'avoir pour regarder les œuvres, parcourir les salles et glaner l'information à sa guise, un minimum de données sur différentes approches des œuvres devrait, dans la même ligne de pensée, être accessible afin que tous s'y sentent suffisamment à l'aise, un peu comme chez eux. C'est ainsi que les différents modes d'apprentissages informels ouverts et non dirigés deviennent réellement accessibles à tous ${ }^{[4]}$, d'où les recherches sur le contenu et la forme d'une pédagogie spécifique du musée que nous essayons de mettre en place. Cette pédagogie devient alors une sorte de manière de vivre et de savoir vivre au sein de chaque musée.

Sous le thème de l'hospitalité, on parle moins de frontières qui séparent l'art et la vie, préférant considérer chaque musée comme un lieu ouvert à tous, chaque œuvre comme un seuil à franchir. Il suffit, et c'est là le défi, de connaître et de savoir pratiquer les " us et coutumes » des œuvres, mais aussi ceux des visiteurs. Notre rôle de médiateur, entre le visiteur et l'art, est d'autant plus riche et complexe, d'autant plus généreux. Hôtes du musée et de maintes démarches artistiques, visions et représentations du monde auxquelles les visiteurs dans toute leur individualité sont conviés, notre rôle est de les aider à rencontrer réellement et librement l'œuvre d'art : physiquement et spirituellement, émotionnellement et intellectuellement. De les accueillir chez nous, chez eux, le musée étant un lieu public où chacun est libre d'imaginer, de rêver, de laisser une partie de lui-même dans l'esprit du musée, dans son histoire. Notre enquête sur l'intégration de la notion d'hospitalité au sein de la diversité que représente le travail de médiation est loin d'être terminée, mais nous sommes convaincus que chacun doit construire pour lui-même son propre principe d'accueil, sa propre définition d'hospitalité pour mener ses visites, pour construire les dispositifs pédagogiques pour son musée, en fonction de ses visiteurs, ou, mieux, de ses hôtes.

\section{[4]}

Les styles d'apprentissage mis en place dans les British Galleries du Victoria and Albert Museum sont un bon exemple que nous étudions. 


\section{Questionnaire proposé aux dix conservateurs,}

chefs d'établissement, à l'automne 2006

En 2007 avec dix musées, comment devrait s'appeler notre service?

Comment ce service devrait-il être structuré?
Service éducatif ou Service des publics ou autre... (spécifier)

- Un seul service: développer/privilégier le travail inter-musées tout en s'appuyant sur les spécificités de.chacun

- ou deux services éducatifs (SE): MAMCS/CTU (Musée d'Art moderne et contemporain / Musée Tomi Ungerer) et pôle musées de patrimoine

- ou trois SE: MAMCS/CTU, musées d'art ancien, musées de société

- ou davantage de SE proprement dits, mais en répartissant le personnel de médiation dans les différents musées, sous la direction des conservateurs, chefs d'établissement
Comment décrivez-vous votre musée... Parmi ces notions, lesquelles (vous limiter à 3 ou 4) souhaiteriez-vous associer à votre musée (vos objectifs)?
- Intégré dans la vie culturelle strasbourgeoise, reconnu nationalement, aura européenne, voire mondiale

- Lieu de rencontre, de divertissement, de débat, de ressources, de recueillement, de recherche, de contemplation, de quiétude, d'érudition, de liberté, de connaissance... autres (spécifier)

- Dynamique, populaire, familial, convivial, précurseur/avant-gardiste, engagé, ouvert, confortable, esthétique... autres (spécifier) 


Pour chacun des cinq points
suivants, si vous aviez à
choisir, quelles seraient
vos priorités?

1] Travailler pour

- Collections

- Expositions

2] Privilégier

- Groupes

- Visiteurs individuels

3] Accueillir

- Scolaires tous niveaux confondus

- Scolaires un(des) niveau(x) particulier(s)

- Enseignants, médiateurs, éducateurs (relais)

- Étudiants

- Familles

- Touristes

- Personnes en situation de handicap

- Personnes en insertion

- Structures de loisirs

- Adultes

- Enfants hors temps scolaire

- Professionnels de la culture, de la médiation

- Officiels (politique, culturel...)

- Entreprises, comités d'entreprise, Communauté urbaine de Strasbourg (CUS)

- Mécènes

- Quartier, public local, Bas-Rhinois, Alsaciens, Français, Allemands, Européens

- Autres (spécifier)

4] Types d'accueil

- Visites scolaires

- Visites "découverte" tous publics

- Ateliers enfants

- Ateliers adultes

- Approches insolites

- Conférences

- Musique dans les salles, lecture de textes ou activités interdisciplinaires

- Rencontres avec des personnalités

- Expositions pédagogiques inter-musées

- Nuits des musées et autres évènements ponctuels

- Autres

5] Aides à la visite

- Cartels allongés

- Fiches salles

- Fiches œuvres, artistes, thématiques

- Audioguides

- Dispositifs interactifs, ludiques

- Information ponctuelle sur le musée

- CD-ROM

- Petits journaux adultes

- Carnets de regard, fiches ludiques, approches pour les enfants

- Parcours thématiques

- Salles d'introductions, d'informations, de ressources...

- Espaces d'expérimentation

- Éditions grand public

- Éditions jeune public

- Dossiers de préparation à la visite

- Formations enseignants, médiateurs, etc.

- Autres 
Au volet de l'organisation, que pensez vous:
- D'une centrale de réservation pour l'ensemble des musées

- De restreindre l'accueil de groupes à des moments précis, à un nombre limité par musée/jour

- De séparer les groupes des visiteurs individuels

- De laisser les groupes visiter librement les musées, de nous concentrer sur les formateurs, les relais, les groupes spéciaux et les aides à la visite

- De privilégier les moments de fermeture du musée pour des visites hors normes / projets spéciaux

- D'augmenter les tarifs d'accueil pour compenser les coûts

- De développer des partenariats qui "feront" à notre place... l'Office de tourisme de Strasbourg (OTS) pour nos visites guidées, les associations comme l'Association pour le Musée d'art moderne et contemporain de Strasbourg (AMAMCS) pour certains accueils... 


\section{Summary}

[Translated by Micheline Giroux-Aubin]

In order to better understand the expectations related to visitors' reception and initiate a discussion on a public policy common to the ten museums of the city of Strasbourg, we proposed (end of 2006) a questionnaire to the conservators and the institution directors. To ascertain that the resulting mismatch between the different conceptions of the museums would be an incentive rather than an obstacle, we retained as a model the famous slogan of Amnesty International, "All different - all equal," where equality is asserted through the very expression of differences. All that was left then was to find a means for the divergent visions of the conservators on their respective museum to become forces that motivate new ways of welcoming and accompanying visitors in each museum. From a thorough analysis of the answers emerged three types of museums that became our operational structure (museums as a space of living, museums as a space of delight, museums as the starting point of committed social approaches). These, together with a mediation philosophy based on the multiple readings of the hospitality notion, helped in reacting to the various situations to which the educative service is confronted in the ten Strasbourg museums when dealing with visitors' reception. 